\title{
Looking for Self-Organized Critical Behavior in Avalanches of Slightly Cohesive Powders
}

\author{
M. A. S. Quintanilla, ${ }^{1}$ J. M. Valverde, ${ }^{1}$ A. Castellanos, ${ }^{1}$ and R. E. Viturro ${ }^{2}$ \\ ${ }^{1}$ Departamento de Electronica y Electromagnetismo, Universidad de Sevilla, \\ Avenida Reina Mercedes s/n, 41012 Sevilla, Spain \\ ${ }^{2}$ Xerox Corporation, 800 Phillips Road, Webster, New York 14580 \\ (Received 15 June 2001; published 18 October 2001)
}

\begin{abstract}
We report results from a statistical analysis of avalanches of cohesive powders in a slowly rotated drum. Interparticle adhesion, which diminishes the effect of inertia and whose magnitude strongly fluctuates in a local scale, makes avalanches in slightly cohesive powders eligible for displaying self-organized criticality. However, the results show that avalanche sizes, time interval between avalanches, and maximum stable angle do not follow a power-law distribution. Otherwise, these parameters scale with powder cohesiveness.
\end{abstract}

DOI: 10.1103/PhysRevLett.87.194301

A large number of extended dissipative dynamical systems are thought to naturally organize themselves into a stationary critical state where transport takes place through events lacking any length and time scales, with fluctuations involving frequency spectra $1 / f^{\beta}(\beta \sim 1)$. This is known as self-organized criticality (SOC); its robustness against any modification of the system is its most essential property. The concept of SOC was first introduced in terms of simple cellular automaton models that could reproduce a $1 / f$ power spectrum [1]. Indeed the power-law characteristic of SOC has been found in the noisy behavior of some transport processes over vastly different time scales as, for instance, the Barkhausen noise of ferromagnets [2], or the distribution of earthquakes' magnitudes [3].

Although granular piles were suggested as a clear example of a self-organized system [1], whether fluctuations in the intermittent flow at the surface of granular materials display SOC or not remains an actively discussed question [4-9]. In a seminal experiment Jaeger et al. [4] found that avalanches generated in a rotating drum were not ruled by SOC. Instead, a peak distribution was observed in the power spectrum due to the existence of a regular interval between the maximum stable angle of the pile and the angle of repose. Held et al. [5] investigated surface avalanches in circular sandpiles resulting from a single local addition of grains to the pile. They reported that, although for large sandpiles the distribution of avalanches became sharply peaked, sufficiently small sandpiles showed SOC with a $1 / f^{2}$ power spectrum. Liu et al. [6] examined how the finite size affected the nature of avalanches and concluded that it masked the observance of a first order transition in very small piles. A further criticism of these works is that the employed techniques could only detect avalanches that slid out of the pile. Bretz et al. [7] solved this problem by applying digital image analysis to record avalanches in a slowly rotating drum. They found that large sliding events occurred with a certain periodicity, whereas small "precursor" avalanches had a power-law size distribution. The number of these small avalanches was shown to have a power-law behavior over time with an exponent of -1
PACS numbers: 45.70.Ht, 05.65.+b

reminiscent of SOC, but it was observed only over one decade. Cantelaube et al. [8] used digital image analysis and found that the size distribution of small discharge events could be described by a power law, whereas the size and time duration of large sliding events were well averaged. They found that the spectrum of the mass fluctuations followed the power law $1 / f^{1.5}$, but again the statistics were not sufficient to confirm it unequivocally. By slowly adding elongated rice grains to a pile, Frette et al. [9] found evidence of SOC behavior on the dynamics of the pile. The absence of SOC for less elongated grains suggested that its occurrence depends on the detailed mechanism of energy dissipation. They argued that a system of anisotropic grains could pack in a large variety of configurations and because of the highly disordered structure each avalanche replaced a configuration with another, different, configuration, giving as a result a "dynamically varying disorder." Moreover the elongation of the particles enhanced the frictional contact and reduced the inertia effects leading to a dynamics dominated by local mechanisms. According to Frette et al. [9] these are requirements for a granular system to display SOC.

Interparticle adhesive forces are extremely important in geophysical applications, such as snow avalanches or soils, and in the transport of fine powders in the chemical industry. In spite of this, most of the efforts made in the search for SOC have been focused on noncohesive dry grains. It is generally observed that the tendency of cohesive particles to aggregate leads to a substantial departure from the behavior of noncohesive grains when the interparticle adhesion force exceeds particle weight [10]. Adhesion leads to the formation of large scale semicoherent granular structures, causing the site of failure to move from the surface (noncohesive grains) into the bulk of the powder (highly cohesive grains) [11]. While adhesion may be due to van der Waals, electrostatic, and capillary attractive forces, van der Waals force is dominant between uncharged and dry fine particles [12]. We have found that adhesion between fine polymer particles, arising from this source, increases markedly with the consolidation stress and time 
of consolidation [13], indicating plastic deformation of the particle contact area. Another important property is that stresses in cohesive powders are highly fluctuating in a local scale [12].

In light of Frette et al.'s arguments, one would think that avalanches in slightly cohesive powders, even with spherical grains, meet the requirements for exhibiting SOC: Powders contains strongly disordered regions of varying degrees of consolidation, whose distribution changes randomly after each avalanche, and the free surface is very rough, yielding a very broad distribution of avalanche sizes. Furthermore particle motions are constrained by adhesion forces, counteracting the effect of inertia and increasing dissipation. In such properties slightly cohesive powders are akin to long-grained rice. We investigate in this Letter whether avalanches in cohesive powders really display SOC by means of analyzing the succession of avalanches of powders of different degrees of cohesiveness in a rotating drum.

The materials used, named "developers," are employed in the xerographic industry [14]. Our developer beads are about $100 \mu \mathrm{m}$ in diameter and are made of a magnetic material with a polymer coating. A monolayer of toner particles $(\sim 10 \mu \mathrm{m}$ polymer particles) is adhered to the beads by triboelectric charge exchange. Cohesiveness of the powder ensemble arises from van der Waals attractive forces between toner particles and increases with time during which the material, in the manufacturing process known as "housing," is subjected to compressive stresses. Measurements of the cohesion of these developers are reported elsewhere [14]. In the range of the cohesiveness we deal with in this Letter, the ratio of interparticle adhesion force to particle weight goes from $\sim 10$ to $\sim 100$. For comparison, noncohesive spherical beads (soda-lime glass), which are 400-600 $\mu \mathrm{m}$ in diameter, have also been tested. In the experiments we used a computer controlled step motor to rotate a polycarbonate drum $(7.4 \mathrm{~cm}$ inner diameter and $2 \mathrm{~cm}$ depth). The drum was rotated at very slow velocities, $0.288^{\circ} / \mathrm{s}$ for developers and $0.044^{\circ} / \mathrm{s}$ for the beads (these velocities were tuned to have a similar time spacing between large events in both systems). The drum was placed on an air table and connected to the motor by an elastic tube to isolate it from any external vibration. Avalanches were recorded with a CCD camera interfaced to a computer and controlled with an image processing software. A $\mathrm{C}++$ edge detection routine computed the average angle of the slope $\alpha_{i}$ for every frame $i$. The criteria for detecting an avalanche was that $\alpha_{i}-\alpha_{i+1}>2^{\circ}$ for developers. This threshold allowed us to detect the smallest avalanches, being at the same time larger that the typical indeterminacy in the measurement of $\alpha_{i}$. For glass beads the threshold was fixed at $0.6^{\circ}$. In that case avalanches involved very small portions of material and the angle of the slope could be measured very precisely since the free surface was always quite regular. When an avalanche was detected the maximum stable angle and the area of the avalanching material (avalanche size) were computed and stored. For each powder the experiment was run for about three days, giving a number of the order of 5000 avalanches. The huge number of events recorded was sufficient to undertake a robust statistical analysis.

Typical outputs for the mean angle of the slope of a cohesive powder and of glass beads are shown in Fig. 1a. For the cohesive powder we observe the occurrence of quasiperiodic large events with a noise due to small ones. For glass beads, avalanches occur quite regularly, with nearly uniform size and time spacing, indicating that such a system does not self-organize to a critical state, as was already found by Jaeger et al. [4]. To find out if the noise measured in the cohesive system (Fig. 1a, upper curve) is indicative of SOC behavior we computed the averaged power spectrum of the time signals (Fig. 1b), showing a striking similarity to the power spectrum for glass beads. There is a sharp peak at low frequencies centered around $f_{0}=1 / T_{0}$, with $T_{0} \simeq 200 \mathrm{~s}$ being the average time interval between major events (dominant period), and a crossover to a $\sim 1 / f^{2}$ power law at high frequencies. A

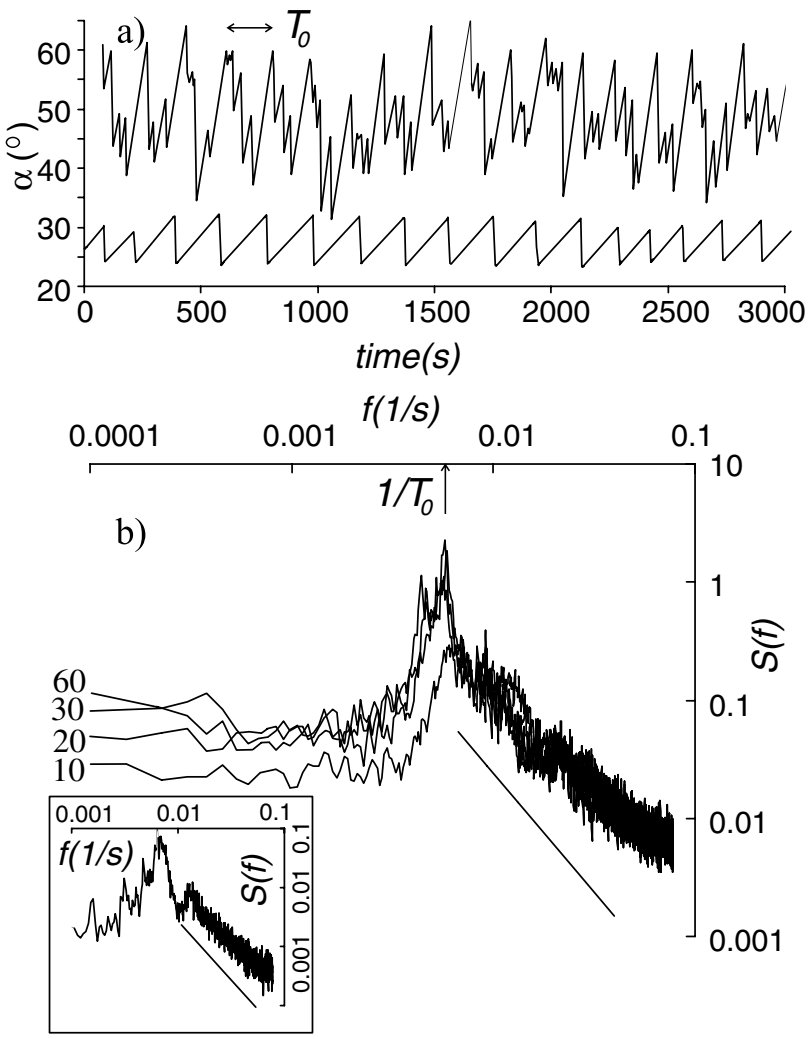

FIG. 1. (a) Typical time evolution of the mean angle of the slope for a cohesive powder (60 min developer, upper curve) and for noncohesive glass beads (lower curve). (b) Power spectrum of the evolution in time of the mean angle of the slope of cohesive powders. The cohesiveness of the powder scales with the housing time which is indicated (here and in the rest of figures) in minutes. The inset shows the power spectrum for noncohesive glass beads. The straight lines indicate $1 / f^{2}$ behavior. 
similar tail is found for the system of glass beads, where avalanches do not display SOC [4], suggesting that the falloff cannot be ascribed to SOC but rather to the finite width of individual avalanches responsible for the sawtooth shape of the signal (a perfect periodic signal of triangular pulses yields a $1 / f^{2}$ tail at high frequencies). The absence of SOC will become evident when we consider the statistics of size of small events.

The probability distribution of avalanche sizes $P(s)$ are plotted in Fig. 2. It can be observed that, although the width of the statistics are broad, $P(s)$ is correlated to cohesion, increasing the average size of avalanches with powder cohesiveness. Moreover the distributions do not show the characteristic power-law tail of critical behavior. In Fig. 3 the probability distributions of the maximum stable angle $P\left(\alpha_{c}\right)$ are shown. The qualitative trend followed by $P\left(\alpha_{c}\right)$ and $P(s)$ with cohesiveness can be analyzed from the insight of continuum theory [11]. A simple model of fracture of a homogeneous cohesive powder yields $1-\cos \left(\alpha_{c}-\phi\right)=4 C \cos \phi /(\rho g D)$ for the maximum stable angle $\alpha_{c}$ of a slope of length $D$, with $\phi$ being the angle of internal friction of the material, $C$ the powder cohesion, $\rho$ the powder bulk density, and $g$ the acceleration due to gravity. The depth of the avalanche $h$ is given by $h / D=[2 C \cos \phi /(\rho g D)]^{1 / 2}$. Thus in noncohesive systems and/or infinite slope limit the maximum stable angle approaches the angle of internal friction, and the depth of the avalanche becomes negligibly small compared to system size. Accordingly we find that the probability distribution of large events decays very rapidly for noncohesive glass beads as compared with the distributions of cohesive powders (Fig. 2). The average avalanche depth for the glass beads is only $\sim 10$ grains, much smaller than the sample depth. Increasing cohesion and/or decreasing the sample size cause an increase of both the maximum stable angle and avalanche depth. In the very high cohesive limit individual particles are so tightly bonded that

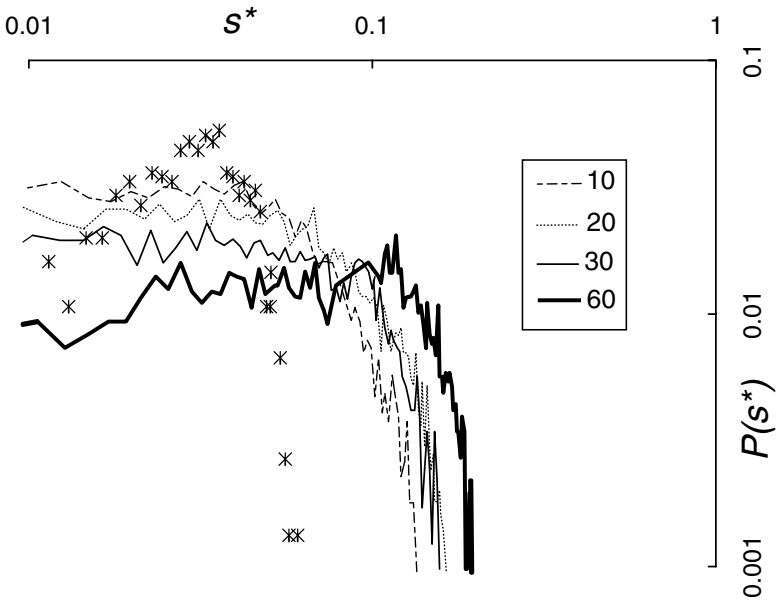

FIG. 2. Probability distribution of the ratio of the avalanche size to the total area covered by the powder $\left(s^{*}\right)$. Symbols represent $P\left(s^{*}\right)$ for glass beads. we would have very large critical angles and avalanche sizes of the order of the sample size. In the slight cohesion range of our powders we find the expected trend: The avalanche size and the maximum stable angle are, on average, increasing functions of cohesiveness (Figs. 2 and 3).

We now analyze the probability distribution of the time interval between the end of an avalanche and the beginning of the next one $[P(\tau)$, Fig. 4]. Note that both $P(\tau)$ and $P\left(\alpha_{c}\right)$ (Fig. 3) are bimodal. As we will see this result is related to the occurrence of small and large events (Fig. 1a and inset of Fig. 4). After a large avalanche a quite irregular slope and a very small average angle of repose are left behind. The powder then takes a long time $\tau_{1}$ to reach a critical state. Once it does, at an angle $\alpha_{c 1}$, and due to the high irregular profile of the free surface, it yields a succession of small precursor avalanches (separated by a short time interval $\tau_{2}$ ) that smooth the surface. The last small event is followed, after a time $\tau \sim \tau_{2}$, by a large event closing the cycle. Accordingly both peaks in $P(\tau)$ should correspond to the averages values of $\tau_{1}$ (buildup time after a large event) and $\tau_{2}$ (buildup time after small precursor event), with $\overline{\tau_{1}}>\overline{\tau_{2}}$. The peaks in $P\left(\alpha_{c}\right)$ should correspond to the averages values of $\alpha_{c 1}$ (angle of the first precursor event) and $\alpha_{c 2}$ (angles of the next precursor events and the large event), with $\overline{\alpha_{c 1}}>\overline{\alpha_{c 2}}$. An inspection of Fig. 1a reveals that, for the $60 \mathrm{~min}$ developer, $\tau_{1}$ is of the order of $100 \mathrm{~s}$, whereas $\tau_{2}$ is mostly below $40 \mathrm{~s}$. Figure 4 indicates that, for this powder, the peaks are centered around $\overline{\tau_{1}} \simeq 95 \mathrm{~s}$ and $\overline{\tau_{2}} \simeq 35 \mathrm{~s}$, thus confirming our interpretation. Furthermore the first precursor avalanche initiates at angles preferentially around $\overline{\alpha_{c 1}} \simeq 60^{\circ}$ and the subsequent events occur usually at angles around $\overline{\alpha_{c 2}} \simeq$ $50^{\circ}$. These values approximately match the angles at which both peaks of $P\left(\alpha_{c}\right)$ are located (Fig. 3). Since the number of small events between major avalanches is

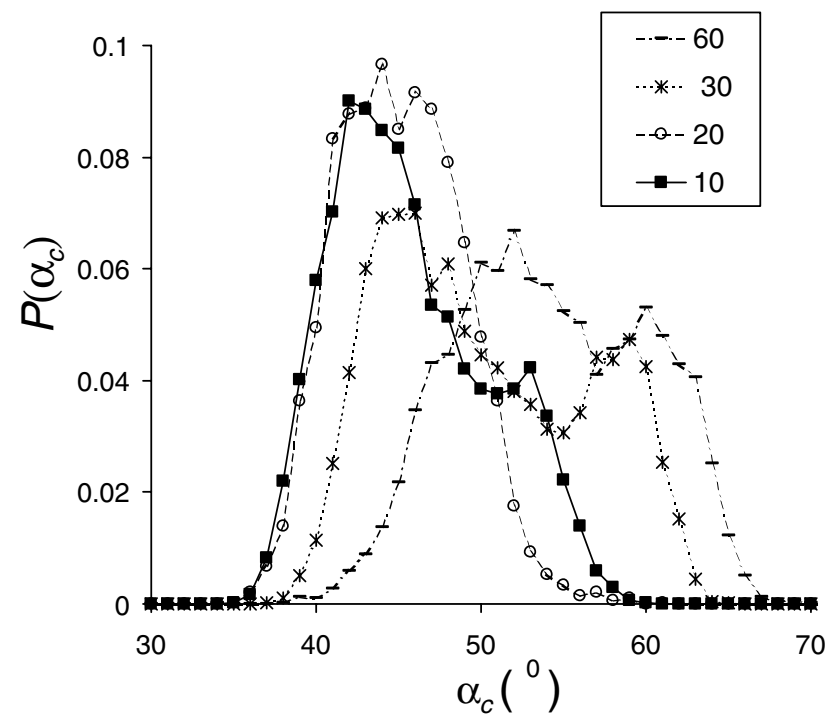

FIG. 3. Probability distribution of the maximum angle of stability $\alpha_{c}$ for the cohesive powders. 


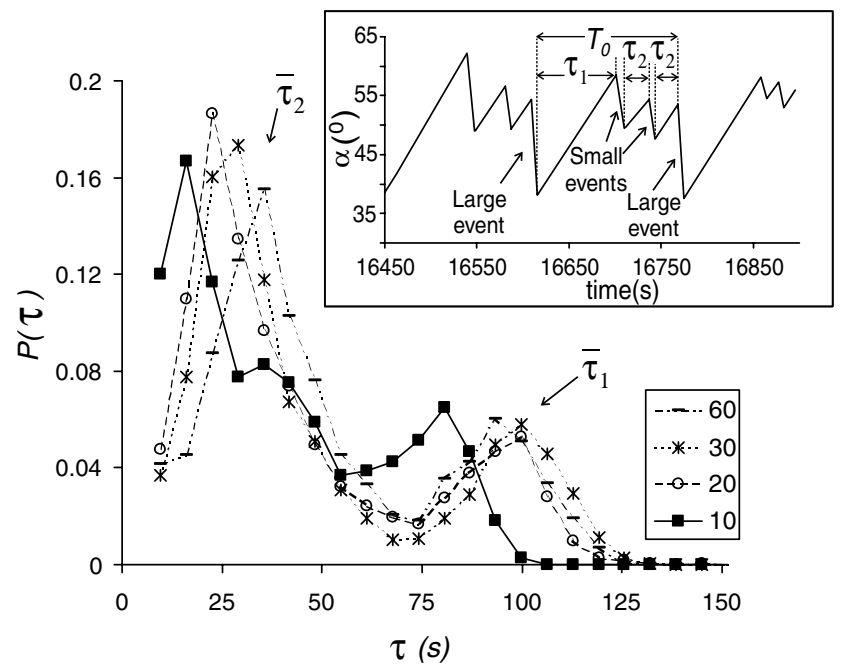

FIG. 4. Probability distribution of time interval $\tau$ between the end of an avalanche and the beginning of the next one for the cohesive powders. The inset is a small sequence of the time evolution of the angle of the slope (Fig. 1a).

around 2 [see Fig. 1a and Fig. 4, where $P\left(\overline{\tau_{2}}\right) \sim 2 P\left(\overline{\tau_{1}}\right)$ ], the time interval between large events (dominant period $T_{0}$ in Fig. 1a) can be estimated as $T_{0} \sim \overline{\tau_{1}}+2 \overline{\tau_{2}} \sim$ $200 \mathrm{~s}$, in agreement with the location of the sharp peak displayed by the frequency spectra of the time series data (Fig. 1b). Finally, the more cohesive the grains are, the larger the average maximum stable angles and therefore the larger $\overline{\tau_{1}}$ and $\overline{\tau_{2}}$ (Fig. 4). However, the effect is not marked since the angle of repose also increases, although slightly, with cohesion.

In conclusion, avalanches of cohesive powders in a rotating drum have been studied in detail and compared to avalanches in a noncohesive granular material. The presence of interparticle adhesive forces causes the site of failure to move from the surface to the interior of the material and reduces inertia. It also adds inhomogeneity to the system which causes some noise in the size and time spacing of avalanches. The power spectrum of the evolution of the angle of the slope shows a crossover from a stretched peak at low frequencies, corresponding to the average time interval between large events, to a power-law tail at high frequencies, reminiscent of SOC behavior. However, the power spectrum of noncohesive grains, where avalanches have a uniform size and time spacing, exhibits the same falloff due to the finite size of the system. The distribution of avalanches sizes, although quite broad, does not show a power-law behavior and is correlated to powder cohesiveness. The distributions of the maximum stable angle and time interval between avalanches present well-defined peaks due to large and small events well averaged in time and critical angle. These peaks are qualitatively correlated to powder cohesiveness as expected from continuum theory. Thus avalanches of slightly cohesive grains do not behave in a self-organized critical manner.

This research has been supported by the Xerox Foundation, Spanish Government Agency Ministerio de Ciencia y Tecnologia (DGES) under Contract No. BMF2000-1056, and NATO Grant LINKAGE PST.CLG.976575.

[1] P. Bak, C. Tang, and K. Wisenfeld, Phys. Rev. Lett. 59, 381 (1987); C. Tang and P. Bak, Phys. Rev. Lett. 60, 2347 (1988).

[2] P. J. Cotel and L. V. Meisel, Phys. Rev. Lett. 67, 1334 (1989).

[3] K. Chen, P. Bak, and S. P. Obukhov, Phys. Rev. A 43, 625 (1991).

[4] H. M. Jaeger, C.-h. Liu, and S. Nagel, Phys. Rev. Lett. 62, 40 (1989).

[5] G. A. Held, D. H. Solina, D. T. Keane, W. J. Haag, P. M. Horn, and G. Grinstein, Phys. Rev. Lett. 65, 1120 (1988).

[6] C.-h. Liu, H. M. Jaeger, and S. R. Nagel, Phys. Rev. A 43, 7091 (1991).

[7] M. Bretz, J. B. Cunningham, P. Kurczyisky, and F. Nori, Phys. Rev. Lett. 69, 2431 (1992).

[8] F. Cantelaube, Y. Limon-Duparcmeur, D. Bideau, and G. H. Ristow, J. Phys. I (France) 5, 581 (1995).

[9] V. Frette, K. Cristensen, A. Malthe-Sorensen, J. Feder, T. Josang, and P. Meakin, Nature (London) 379, 49 (1996).

[10] S. T. Nase, W. L. Vargas, A. A. Abatan, and J. J. McCarthy, Powder Technol. 116, 214 (2001).

[11] J. M. Valverde, A. Castellanos, A. Ramos, and P. K. Watson, Phys. Rev. E 62, 6851 (2000).

[12] M. A. S. Quintanilla, A. Castellanos, and J. M. Valverde, Phys. Rev. E 64, 031301 (2001).

[13] J. M. Valverde, A. Ramos, A. Castellanos, and P. K. Watson, Powder Technol. 97, 237 (1998).

[14] F. C. Genovesse, P. K. Watson, A. Castellanos, and A. Ramos, Powders \& Grains 97, edited by R. P. Behringer and J. Jenkins (Balkema, Rotterdam, 1997), p. 151. 